\title{
Medizinische Versorgung im Alter - welche Evidenz brauchen wir? Empfehlungen der Leopoldina
}

Gute medizinische Versorgung mit hoher Evidenzbasierung ist für viele Menschen mit unterschiedlichsten Erkrankungen in Deutschland möglich. Jedoch ist dies für die Gruppe der betagten und hochbetagten Patienten keine Selbstverständlichkeit im Kontrast zu deren hohen Inanspruchnahme von Gesundheitsleistungen: Pharmaka, operative und interventionelle Eingriffe, pflegerische Versorgung bis hin zur Institutionalisierung. Gegenstand der Stellungnahme unter Federführung der Nationalen Akademie der Wissenschaften Leopoldina war die Beschreibung von Evidenzlücken für betagte Menschen sowie die Erarbeitung von Lösungsansätzen zur Evidenzgenerierung für diesen wachsenden Anteil der Bevölkerung [1].

Betrachtet man die medikamentöse Therapie, so liegt die meiste Evidenz aus Studien vor, die meist an bis zu 65-Jährigen, gelegentlich 70-Jährigen, durchgeführt werden [2, 3]. Diese Studien sind beispielsweise Voraussetzungen für die Zulassung von Medikamenten, deren Risiko/Nutzenprofil möglicherweise im Alter anders ausfällt als bei jüngeren Populationen. Die physiologischen Veränderungen führen einerseits dazu, dass die Pharmakokinetik vieler Wirkstoffe verändert ist. Meist führen eine verminderte renale und teilweise auch reduzierte hepatische Funktionseinschränkung zu einer verlangsamten Elimination. Andererseits kommt es zu Veränderungen an den Zielorganen. Bei herabgesetzter Rezeptordichte können manche Wirkstoffe nicht zur Entfaltung kommen; meist ist jedoch die Sensitivität erhöht, und es kommt zu einer verstärkten Wirkung [3]. Aus den o.g. Studien werden auch Leitlinien entwickelt, von deren Inhalten es oftmals nicht bekannt ist, ob sie auf Betagte übertragen werden können, oder man sieht im klinischen Alltag, dass Medikamente und deren Kombinationen nicht vertragen werden [4].

Daraus lassen sich nicht nur für die Arzneistoffentwicklung und Zulassung Forderungen entwickeln. Diese umfassen den vermehrten Einschluss von betagten und hochbetagten Patienten in Studien, v.a. wenn das Medikament vermutlich häufig in dieser Altersklasse eingesetzt wird. Auch im Alter werden jedoch viele Medikamente zur Primär- und Sekundärprävention eingesetzt; hier werden gerade für alte Patienten relevante Aspekte wie z.B. Funktionalität und Lebensqualität, die Fähigkeit zur Teilhabe am sozialen Leben, bisher wenig bis kaum beachtet. Hinzu kommt die Fokussierung vieler Studien auf eine Erkrankung und ein Medikament, während für viele ältere Menschen die Multimorbidität und das Zusammenspiel ihrer Beschwerden im Vordergrund steht. Aktivitäten des täglichen Lebens und kognitive Fähigkeiten, die die Teilhabe am sozialen Leben ermöglichen, sind bedeutender als Blutdruckwerte und Cholesterinspiegel [5]. Im Rahmen des PREDICT-Projektes wurden unter Einbeziehung von Patienten deren Wünsche und Forderungen an die Forschung untersucht: alte Menschen wollen teilhaben an der Forschung und am Fortschritt, jedoch unter Berücksichtigung ihrer Möglichkeiten und Präferenzen [6].

Ein erhebliches Problem bei der Bewertung von Studien mit älteren Menschen stellt die erhebliche Streuung des allgemeinen Gesundheitszustandes, der körperlichen und der geistigen Funktionen dar. Das kalendarische Alter ist nicht adäquat, die in eine Studie eingeschlossene Patientenpopulation zu beschreiben; vielmehr wird ein geriatrisches Assessment für die entsprechenden Patienten im Rahmen klinischer Studien gefordert [7]. Während in Arzneimittelstudien die Wirksamkeit und Sicherheit neuer Arzneimittel mit hohem finanziellen Einsatz geprüft werden, fehlt es an Studien, die das „DePrescribing“ [8] untersuchen, und die Frage beantworten: Wann ist weniger mehr?

Vergleichbare Forderungen sind an Medizinprodukte zu stellen; die Anwendbarkeit im Alter oder auch die Möglichkeiten des „ambient assisted living“ bieten Chancen für betagte Menschen.

Betagte Patienten haben viele Kontakte mit ihrem Hausarzt, die sich als Behand- lungsanlässe widerspiegeln. Im Gesundheitswesen werden diese wiederum als Diagnosen kodiert, was der Realität oftmals nicht nahe kommt. Die eigentlichen Symptome werden mit der International Classification of Primary Care (ICPC) viel realistischer abgebildet, jedoch nicht vergütet. Auch die WHO-Klassifikation von Funktionsfähigkeit, Behinderung und Gesundheit (ICF) verwendet ein erweitertes Verständnis der Gesundheit, welches den alten Menschen besser beschreibt. Im stationären Sektor muss die typische Krankenhausaufnahmediagnose „reduzierter Allgemeinzustand“ auf ein Organsystem, wie z.B. Nierenversagen, Infektitionskrankheit o.ä., reduziert werden. An dieser Stelle zeigt sich, dass im Gesundheitssystem umgedacht werden müsste, um eine Versorgungsrealität, orientiert an den Bedürfnissen alter Menschen, zu ermöglichen und zu vergüten. Hierzu dient beispielsweise das Chronic Care Modell, welches eine regelmäßige und interprofessionelle Versorgung multimorbider Patienten beinhaltet, um ein Abgleiten in einen schlechteren Zustand zu verhindern. Ein Disease Management Programm Multimorbidität könnte einen sinnvollen Ansatz darstellen.

Das Überschreiten der Sektorengrenzen sowohl ambulant-stationär als auch „zurück in die Häuslichkeit" stellt für geriatrische Patienten eine brisante Situation dar, verbunden mit vielerlei Gefahren [9]. Schon in der Notaufnahme fehlt es an geriatrisch geschultem Personal sowohl im ärztlichen als auch pflegerischen Bereich. Geriatrisches Know-how ist auf den meisten akut ausgerichteten Stationen nicht verfügbar, obwohl schon die fremde Umgebung für multimorbide Senioren ein hohes Risiko für das Entstehen eines Delirs und weiterer Folgekomplikationen darstellt. Trotz des gesetzlich vorgeschriebenen Überleitungsmanagements entstehen Versorgungsbrüche, die zu verzögertem Heilungsverlauf beitragen. Auf diesem Feld besteht noch Forschungsbedarf, und es mangelt an der Umsetzung bereits bekannt nützlicher Konzepte. In der Situation der stationären Entlassung bei noch unzu- 
reichenden Alltagskompetenzen ergibt sich allzu oft eine Kurzzeitpflege, die fast schon als Einbahnstraße gesehen werden kann und unweigerlich in die stationäre Altenpflege führt.

Als Kernthemen werden in der Stellungnahme der Leopoldina das Therapieren „gegen“ bestehende Leitlinien (aufgrund der mangelnden Validität für Multimorbidität) und Shared Decision Making aufgeführt. Eine Evidenz-basierte Medizin muss auch für den zunehmenden Anteil betagter Menschen möglich sein. Dies erfordert neue Anforderungen an Zulassungsstudien für Arzneimittel und Medizinprodukte und v. a. die angemessene Berücksichtigung des Alters und der Multimorbidität in Leitlinien der Fachgesellschaften. Nicht nur Arzneimittel und Medizinprodukte, sondern auch Interventionen wie Verhaltenstherapie sowie komplexe Interventionen im Versorgungsablauf müssen für betagte Menschen evaluiert werden, um eine gesicherte Grundlage für Veränderungen im Gesundheitswesen zu generieren. Hier wird eine besondere Rolle der öffentlichen Förderung (DFG, BMBF, Innovationsfonds) gesehen. Auch diese Forschung muss passgenau zu den Präferenzen alter Menschen sein; das bedeutet auch andere Studienendpunkte als bisher. Last but not least müssen diese Erkenntnisse in die Aus-, Fort- und Weiterbildung einfließen.

\section{Interessenkonflikt}

Die Autorin gibt an, dass kein Interessenkonflikt besteht.

\section{Autorinnen/Autoren}

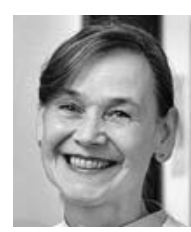

\section{Petra A. Thürmann}

Prof. Dr. med., Lehrstuhl für Klinische Pharmakologie, Universität Witten/Herdecke, Helios Universitätsklinikum Wuppertal
Korrespondenzadresse

Prof. Dr. med. Petra A. Thürmann Lehrstuhl für Klinische Pharmakologie Universität Witten/Herdecke

Helios Universitätsklinikum Wuppertal Heusnerstraße 40

42283 Wuppertal

petra.thuermann@helios-gesundheit.de

Literatur

[1] Nationale Akademie der Wissenschaften Leopoldina; acatech - Deutsche Akademie der Technikwissenschaften; Union der deutschen Akademien der Wissenschaften. Medizinische Versorgung im Alter - Welche Evidenz brauchen wir? Halle (Saale) September 2015. Im Internet: https://www.akademienunion. de/fileadmin/redaktion/user_upload/ Publikationen/Stellungnahmen/3Akad Stellungnahme_Evidenzbasierung_2015_ web.pdf; Stand: 24.09.2019

[2] Downing NS, Shah ND, Neiman JH et al. Participation of the elderly, women, and minorities in pivotal trials supporting 20112013 U.S. Food and Drug Administration approvals. Trials 2016; 17: 199. doi:10.1186/ s13063-016-1322-4 